\title{
Ultrasonic melt treatment in a DC casting launder: The role of melt processing temperature
}

\author{
Christopher Beckwith ${ }^{a^{*}}$, Tungky Subroto ${ }^{b}$, Koulis Pericleous ${ }^{a}$ Georgi Djambazov ${ }^{a}$, Dmitry G. Eskin ${ }^{b, c}$, lakovos \\ Tzanakis d.e \\ ${ }^{a}$ Computational Science and Engineering Group, University of Greenwich, 30 Park Row, London SE10 9LS, UK \\ ${ }^{b}$ Brunel Centre for Advanced Solidification Technology (BCAST), Brunel University London, Uxbridge, UB8 3PH, \\ UK \\ 'Tomsk State University, Tomsk, 634050, Russia \\ ${ }^{\mathrm{d}}$ Faculty of Technology, Design and Environment, Oxford Brookes University, Oxford, OX33 1HX, UK \\ eDepartment of Materials, University of Oxford, Oxford, OX1 3PH, United Kingdom
}

*) Corresponding author, e-mail address: c.beckwith@greenwich.ac.uk

Ultrasonic melt treatment (UST) using a single sonotrode source in a launder is an efficient way to treat a largevolume melt. One key parameter is the melt processing temperature. Melt processing temperature affects the acoustic pressure generated by the sonotrode, which ultimately defines the cavitation development as well as the resulting acoustic streaming. Experimental results also show that processing temperature affects intermetallic number density and the final grain size. This work presents a numerical model covering acoustic cavitation, flow (including acoustic streaming) and heat transfer in direct-chill (DC) casting, to better understand this process. The UST effectiveness is quantified through the size of the high-pressure acoustic region and the melt residence time, a result reflected in experimental grain size data. The output of this work is useful for optimizing the selection of process parameters for UST DC casting.

Keywords: Ultrasonic melt treatment, cavitation, DC casting, melt temperature, fluid flow, experimental validation

\section{Introduction}

Ultrasonic melt treatment (UST) is an attractive melt processing technique which offers an economical and environmentally friendly solution to improve the quality of aluminum alloy billets produced through direct chill (DC) casting. An advantage to UST is that it requires a smaller quantity of costly grain refiner to achieve a similar billet quality. Structure refinement mechanisms by UST include the activation of non-metallic inclusions (e.g. oxides), and fragmentation of intermetallics and Al-dendrites [1, 2]. Although UST has been trialed for decades to treat aluminum alloys in small-scale solidification experiments, upscaling has required the use of multiple sonotrodes. Implementation of this process to perform an effective large-scale melt treatment using a single sonotrode setup is an ongoing challenge. In order to achieve this, the current strategy for upscaling and improving cost effectiveness is to perform UST in the DC casting launder instead of the hot top where UST is traditionally carried out $[1,3,4]$.

When the UST is performed in the molten state of an alloy, the main structure refinement mechanisms differ from when the alloy is treated nearby the solidification front. The main refinement mechanisms are enhanced heterogeneous nucleation through the activation of non-metallic inclusions for the former regime and the fragmentation of primary intermetallics for the latter. It has been shown that the addition of $\mathrm{Zr}$ above its solubility limit in the liquid $\mathrm{Al}$ can refine grain structure of $\mathrm{Al}$ alloys [5]. The $\mathrm{Al}_{3} \mathrm{Zr}$ phase has a small crystallographic misorientation with the Al-matrix [5], thus it has a strong potential to act as a substrate where $\mathrm{Al}$ grains can initiate and grow. Meanwhile, Ti is also a known grain refiner for $\mathrm{Al}$ alloys through either nucleation or growth restriction mechanisms [6] Previous works in UST in aluminum alloys revealed that the combination of $\mathrm{Zr}$ addition above its solubility limit with a small amount of Ti can result in a significant structure refinement $[7,8]$. It has been explained that $\mathrm{Al}_{3} \mathrm{Zr}$ then become the main actor for Al-grain initiation points through enhanced heterogeneous nucleation [9], while the presence of free Ti in the alloy may acts as an effective growth restrictor of aluminum grains [10]. For the fragmentation of primary intermetallics (i.e. $\mathrm{Al}_{3} \mathrm{Zr}$ ), the principal mechanism is the collapse of cavitation bubbles due to fluctuations induced by the vibrating sonotrode. Upon collapse, cavitation bubbles generate high pressure shockwaves which have been shown to be sufficient to trigger the fragmentation [11]. 
Note that both mechanisms likely depend on the UST treatment temperature. Wang et al. [12] found that the most optimum treatment temperature in terms of produced $\mathrm{Al}_{3} \mathrm{Zr}$ crystals size is across the liquidus of this primary phase. In addition, changes to melt properties such as the speed of sound, surface tension, viscosity and hydrogen solubility will lead to different acoustic pressures, and different levels of cavitation activity which in turn will change the rate of occurrence of collapsing events, and thus shockwaves, which drive the fragmentation.

A nonlinear Helmholtz model with terms accounting for the attenuation and the speed of sound variation caused by the existence of cavitating bubbles has previously been used for such problems [3, 13, 14]. In [15], an updated model was suggested that used the effective wavenumber of the bubbly liquid along with density variations which could be due to temperature or the simulation of multiple phases. The latest model includes acoustic streaming, cavitation phenomenon and can simulate the acoustic pressure in the launder. This model has been utilized in a pilot-scale DC casting launder for a water analogue system [15] and has been used for the optimization of various UST-DC casting parameters, such as the inlet flow rate and flow management configurations (in form of partitions). The primary aim of this model is to optimize the residence time (the duration the liquid alloy spends in the high acoustic pressure region of intense cavitation activity), as it has been reported that longer treatment links to better treatment quality [12]. In addition, acoustic resonance might also play a role in treatment quality [17] due to the formation of secondary, though less powerful, treatment zones.

The previous version of this model did not include the effect of heat extraction in the melt by its surroundings during the transfer from the furnace to the hot top. It also did not include the effect of different hydrogen levels at different treatment temperature on the resulting residence time and acoustic pressure distribution. These gaps have been bridged in this work. The predicted effect from these results provide a potential explanation for some of the microstructure differences observed in experiments. The outcome from this work acts as an intermediate step towards a more comprehensive UST-DC casting model which could be used to optimize UST-DC-casting process at an industrial scale.

\section{Materials and methods}

A pilot-scale DC caster with a hot top set up which can produce 152-mm diameter billets was utilized for our experiments performed at Advanced Metal Casting Centre at Brunel University of London. Two billets were cast with a casting speed of $140 \mathrm{~mm} / \mathrm{min}$; one at $923 \mathrm{~K}$ and the other at $938 \mathrm{~K}$ (temperature measured at the inlet to the hot top). A customized AA6XXX-series aluminum alloy without AlTiB grain refiner was used as a base alloy with $0.2 \mathrm{wt} \% \mathrm{Zr}$ added and solidification path for such an alloy was calculated using Thermocalc software (TCAL4 database): the liquidus temperature of $\mathrm{Al}_{3} \mathrm{Zr}$ was $1006 \mathrm{~K}$ while the $\alpha$-Aluminum started to form at around $924 \mathrm{~K}$. The temperature was controlled by a K-thermocouple placed in the hot top. A DC-casting launder was used to transfer the melt from the furnace to the DC-casting hot top. More information regarding the DCcasting set up can be found elsewhere [18].

UST was performed in the DC-casting launder using a water-cooled 5-kW magnetostrictive transducer (Reltec) operating at $17.3 \mathrm{kHz}$ with the power set to $3.5 \mathrm{~kW}$ (approx. $40 \mu \mathrm{m}$ peak-to-peak amplitude). The treatment was performed with a $\mathrm{Nb}$ conical sonotrode $(20 \mathrm{~mm}$ in diameter) immersed approximately $12 \mathrm{~mm}$ below the melt surface and approximately $250 \mathrm{~mm}$ upstream from DC-casting hot top. The sonotrode remained submerged in the melt flow throughout the entire casting process.

For microstructure observations, samples were obtained from the center part, mid-radius, and surface of the billet. Samples were mechanically ground, polished, and then examined under a light optical microscope (Zeiss Axio Scope.A1). For grain size analysis, the polished samples were subsequently anodized using Barker's solution (5\% HBF4 in water solution) for approximately $1 \mathrm{~min}$ at $20 \mathrm{VDC}$. Grain size analysis was performed using the linear intercept method to obtain statistical soundness. Particle number density was calculated by counting the number of lines that intercept with $\mathrm{Al}_{3} \mathrm{Zr}$ intermetallics, divided by the total number of lines used in the analysis of each condition.

\section{Mathematical model}

Assuming the acoustic field in a cavitating bubbly liquid can approximated by its harmonic component, the complex amplitude P can be described by a Helmholtz type model [13, 14, 18]. In previous work [15] this was extended to consider density variations, which are considered here to be due to changes in temperature (see Table 1). Other causes of density variation, for example due to changes in the bubble fraction, are neglected. 
The Helmholtz model used in this work is given by Equation (1). with a nonlinear modified squared wavenumber $k_{m}^{2}$ given by Equation (2).

$$
\begin{aligned}
& \nabla\left(\frac{1}{\rho} \nabla P\right)+\frac{k_{m}^{2}}{\rho} P=0 \\
& k_{m}^{2}=\left(\frac{\omega}{c}\right)^{2}-\frac{\mathcal{A}(P)}{|P|}-i \frac{\mathcal{B}(P)}{|P|}
\end{aligned}
$$

The dissipation functions $\mathcal{A}$ and $\mathcal{B}$ derived in [13], describe the change in the speed of sound and attenuation due to the existence of bubbles.

$$
\mathcal{A}=-\frac{\rho_{l} \omega^{2}}{\pi} \int_{0}^{2 \pi} \frac{\partial \beta}{\partial \tau} \sin \tau d \tau, \quad \mathcal{B}=-\frac{\rho_{l} \omega^{2}}{\pi} \int_{0}^{2 \pi} \frac{\partial \beta}{\partial \tau} \cos \tau d \tau
$$

Where $\tau$ denotes one time period scaled between $[0,2 \pi], \beta=(4 / 3) \pi R^{3} N$ is the void fraction, $R$ is the radius of a bubble, and $N$ is the quantity of hydrogen bubbles per unit volume. To calculate the variation in $R$ over one time period, a bubble dynamics simulation must first be carried out. The Keller-Miksis (KM) equation [19] was used as it contains terms which account for liquid compressibility and acoustic radiation. The Julia DifferentialEquations.jl package [20] was used to solve the ODE given in Equation (4) and an ensemble problem was set up to simulate the bubble behavior at different temperatures. The material properties at each temperature were estimated with the values in Table 1.

$$
\left(1-\frac{\dot{R}}{c}\right) R \ddot{R}+\frac{3}{2} \dot{R}^{2}\left(1-\frac{\dot{R}}{3 c}\right)=\frac{1}{\rho_{l}}\left(1+\frac{\dot{R}}{c}+\frac{R}{c} \frac{d}{d t}\right)\left[p_{l}-p(t)\right]
$$

Where the pressure term $p(t)=p_{0}(1-A \sin (\omega t))$ accounts for background atmospheric pressure, and the sinusoidal acoustic pressure with dimensionless amplitude $A$. The liquid pressure at the liquid-gas interface, $p_{l}$ is defined by Equation (5)

$$
p_{l}=p_{g}-\frac{2 \sigma}{R}-\frac{4 \mu \dot{R}}{R}
$$

Where $\sigma$ is the surface tension, $\mu$ is the liquid viscosity, and $p_{g}$ is the pressure in the gas at the interface, which can be assumed to follow the equation of state [21]:

$$
\dot{p_{g}}=\frac{3}{R}\left[\left.(\gamma-1)\left(\kappa \frac{\partial T}{\partial r}\right)\right|_{r=R}-\gamma p_{g} \dot{R}\right]
$$

where $\gamma=1.4$ is the polytropic exponent, and $p_{g 0}$ the initial gas pressure in the bubble. In previous work [3, 18], a value of $A=2.4$ was chosen to match with the expected pressure under the sonotrode observed in experiments involving the processing of liquid aluminium, but for this work $A$ was taken to be the local pressure calculated by the Helmholtz equation, and the relevant coefficients $\mathcal{A}$ and $\mathcal{B}$ were chosen throughout the domain. This change ensures that the attenuation and speed of sound variation is not underestimated at high pressures $\left(|P|>1.5 P_{\text {blake }}\right)$ or overestimated at lower pressures.

Temperature dependence in the Helmholtz model (1) is introduced by varying the number of hydrogen bubbles per unit volume, $N$. Physically, the volume of hydrogen has been observed to change with temperature due to changes in the solubility of hydrogen, and changes in the efficiency of degassing at different temperature. Data obtained from ultrasonic degassing experiments in a $0.2 \mathrm{~kg}$ crucible with temperatures between $893 \mathrm{~K}$ and 1013 $\mathrm{K}$ [22] show that with an increase in processing temperature, the degassing of the melt increases due to lower viscosity making it easier for bubbles to coagulate and float. In addition, the one-way rectified diffusion coefficient of hydrogen from the liquid phase into the oscillating bubble increases with increasing temperature, increasing the growth rate of the bubble allowing it to float to the surface more easily. Using this data, and assuming $60 \%$ humidity a function $\phi$ was used to represent the hydrogen gas volume per unit volume as a function of temperature and is shown in Fig 1a. The number of bubbles can then be calculated by $N=$ $3 \phi / 4 \pi R^{3}$. 

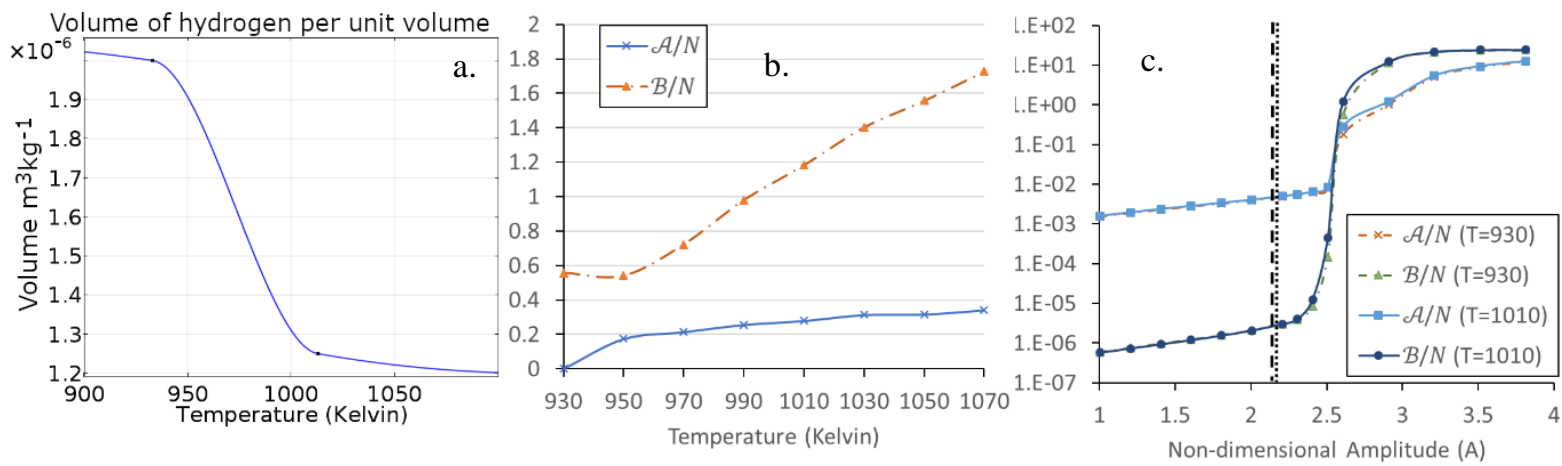

Figure 1. (a) The estimated volume of hydrogen bubbles per unit volume liquid as a function of temperature, using data from [22]. (b) The variation in liquid aluminum attenuation coefficients $\mathcal{A}$ and $\mathcal{B}$ scaled per bubble as a function of temperature at a constant $|P|=2.6 p_{0}$. (c) The same, but as a function of nondimensional pressure amplitude $A=|P| / p_{0}$ at 2 chosen temperatures $(930$ and $1010 \mathrm{~K})$ with their Blake thresholds represented by a black dashed line $(1010 \mathrm{~K})$ and a grey dotted line $(930 \mathrm{~K})$.

Figures $1 \mathrm{~b}$ and $1 \mathrm{c}$ show the effect of temperature and sinusoidal acoustic pressure on the attenuation coefficients $\mathcal{A}$ and $\mathcal{B}$ scaled per bubble. Below the Blake threshold (approximately $|P|=2.18 p_{0}$ at $1010 \mathrm{~K}$ and $|P|=2.2 p_{0}$ at $930 \mathrm{~K})$ and significantly above the where the attenuation coefficients saturate $\left(|P|>3.3 p_{0}\right)$, there is little variation, however the location of the transition region is shifted slightly due to the shift in the Blake threshold, with higher rates of attenuation occurring at higher temperatures, primarily due to decreased viscosity and surface tension. The increase in attenuation can be thought of as an increase in energy loss due to viscous and thermal dissipation, as well as acoustic radiation [13].

Table 1Material properties of liquid aluminum used in the numerical simulation. Data from [23].

\begin{tabular}{|l|l|l|}
\hline Property & Unit & Function \\
\hline Speed of sound, $\mathrm{c}$ & $\mathrm{m} \mathrm{s}^{-1}$ & $4389.39+0.31 \mathrm{~T}$ \\
\hline Density, $\rho$ & $\mathrm{kg} \mathrm{m}^{-3}$ & $2670-0.299 \mathrm{~T}$ \\
\hline Thermal Conductivity, $k$ & $\mathrm{~W} \mathrm{~m}^{-1} \mathrm{k}^{-1}$ & $33.9+0.07982 \mathrm{~T}-2 \mathrm{E}-5 \times \mathrm{T}^{2}$ \\
\hline Dynamic Viscosity, $\mu$ & $\mathrm{Pa} \mathrm{s}^{-1}$ & $0.002955-1.66667 \mathrm{E}-6 \times \mathrm{T}$ \\
\hline Surface Tension, $\sigma$ & $\mathrm{N} \mathrm{m}^{-1}$ & $0.993-0.000127 \mathrm{~T}$ \\
\hline
\end{tabular}

The fluid flow simulation used the steady state turbulent flow solver in COMSOL Multiphysics, using a $k-\omega$ turbulence model, coupled with the heat transfer module. Density variations were handled by an incompressible Boussinesq approximation. The acoustic coupling was treated as an additional acoustic streaming body force $\boldsymbol{F}=-\nabla\left(\rho_{l} \boldsymbol{v} \otimes \boldsymbol{v}\right)$, where $\boldsymbol{v}=\nabla P / \rho \omega$ is the acoustic velocity. The coupled set of fluid flow and heat transfer equations are given by Equations (7)-(9), where $\boldsymbol{u}$ represents the fluid velocity, and $C_{p}$ is the specific heat capacity at constant temperature.

$$
\begin{aligned}
& (\boldsymbol{u} \cdot \nabla) \boldsymbol{u}=-\frac{1}{\rho} \nabla \mathrm{p}+\nu \nabla^{2} \boldsymbol{u}+\boldsymbol{F}+\beta\left(T-T_{0}\right) \boldsymbol{g} \\
& \rho \nabla \cdot(\boldsymbol{u})=0 \\
& \rho C_{p} \boldsymbol{u} \cdot \nabla T=k \nabla^{2} \mathrm{~T}
\end{aligned}
$$

In the previous work [15], a massless particle model was used to calculate the residence time. While this might be efficient, it is a simplistic model which does not account for particle interactions, turbulence, or other effects. Here, a first order Newtonian formulation was used, with a uniform particle size. The particle density changes depending on temperature using the equation in Table 1. Turbulence is added to the model using a discrete random walk.

$$
\frac{d\left(m_{p} \mathbf{v}_{p}\right)}{d t}=\left(m_{p} / \tau_{p}\right)\left(\mathbf{u}-\mathbf{v}_{p}\right)
$$

Where $\tau_{p}$ is the particle velocity response time, $m_{p}$ is the mass of a particle, and $\mathbf{v}_{p}$ the particle velocity. 


\section{Numerical results}

The model described in the Section 3 was used to model the coupled acoustic pressure field, fluid flow, and heat transfer in the DC casting launder where UST was applied. A full summary of boundary conditions for the simulated cases, as well as parameter sets for the sonotrode displacement and inlet temperatures, are given in Table 2 .

Table 2 Boundary conditions

\begin{tabular}{|c|c|}
\hline \multicolumn{2}{|c|}{ Partitions, Sonotrode Wall, Launder } \\
\hline$P$ & Normal gradient $=0$ \\
\hline $\mathbf{u}$ & No slip \\
\hline$T$ & Zero heat flux (Normal gradient $=0$ ) \\
\hline \multicolumn{2}{|l|}{ Sonotrode } \\
\hline$P$ & $\begin{array}{l}\text { Normal gradient }=\omega^{2} \rho \delta / 2 \text { where } \delta \text { represents the peak-to-peak displacement }(20 \mu \mathrm{m} \text { or } \\
40 \mu \mathrm{m} \text { depending on the case) }\end{array}$ \\
\hline $\mathbf{u}$ & No slip \\
\hline$T$ & Zero heat flux (Normal gradient $=0$ ) \\
\hline \multicolumn{2}{|c|}{ Free Surface } \\
\hline$P$ & $P=0$ \\
\hline $\mathbf{u}$ & Slip \\
\hline$T$ & Heat transfer coefficient $\left(46 \mathrm{~W} \mathrm{~m}^{-2} \mathrm{~K}^{-1}\right)$, external temperature $293 \mathrm{~K}$. \\
\hline \multicolumn{2}{|r|}{ ( } \\
\hline$P$ & Nonreflecting (PML) \\
\hline $\mathbf{u}$ & Mass flux \\
\hline$T$ & Fixed inlet temperature ( $950 \mathrm{~K}, 965 \mathrm{~K}, 980 \mathrm{~K}$, and $1040 \mathrm{~K}$ depending on the case) \\
\hline \multicolumn{2}{|l|}{ Outlet } \\
\hline$P$ & Nonreflecting (PML) \\
\hline $\mathbf{u}$ & Pressure outlet \\
\hline$T$ & Heat outflow \\
\hline
\end{tabular}

In 2D simulations of the DC casting launder, the acoustic pressure increases with increasing temperature, up to approximately $1040 \mathrm{~K}$. This also results in an increase in the size of the processing region, which could lead to a marginal increase in the residence time, allowing higher likelihood of particle fragmentation. This change is shown by the highlighted region in Figure 2, for the $20 \mu \mathrm{m}$ amplitude and for $950 \mathrm{~K}$ and $980 \mathrm{~K}$ inlet temperature cases. This region enlargement is partially driven by the pressure increase, but also by the reduction of the Blake threshold at higher temperatures. When using a $40 \mu \mathrm{m}$ sonotrode displacement, significantly higher pressures were predicted under the sonotrode, increasing from approximately $350 \mathrm{kPa}$ up to $900 \mathrm{kPa}$ in a thin layer when compared to the $20 \mu \mathrm{m}$ case. This pressure is attenuated due to increased shielding and causes more intense acoustic streaming.

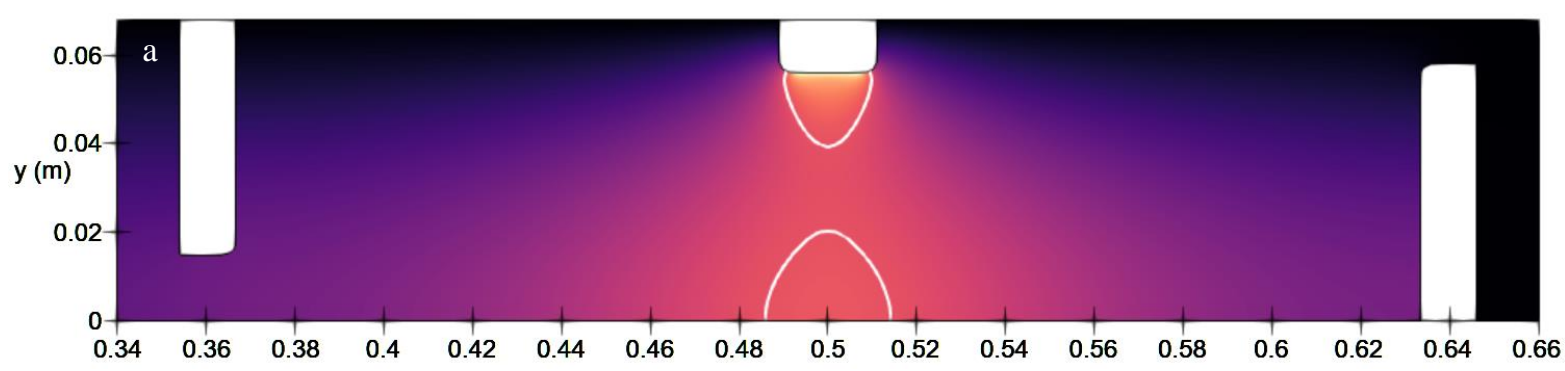




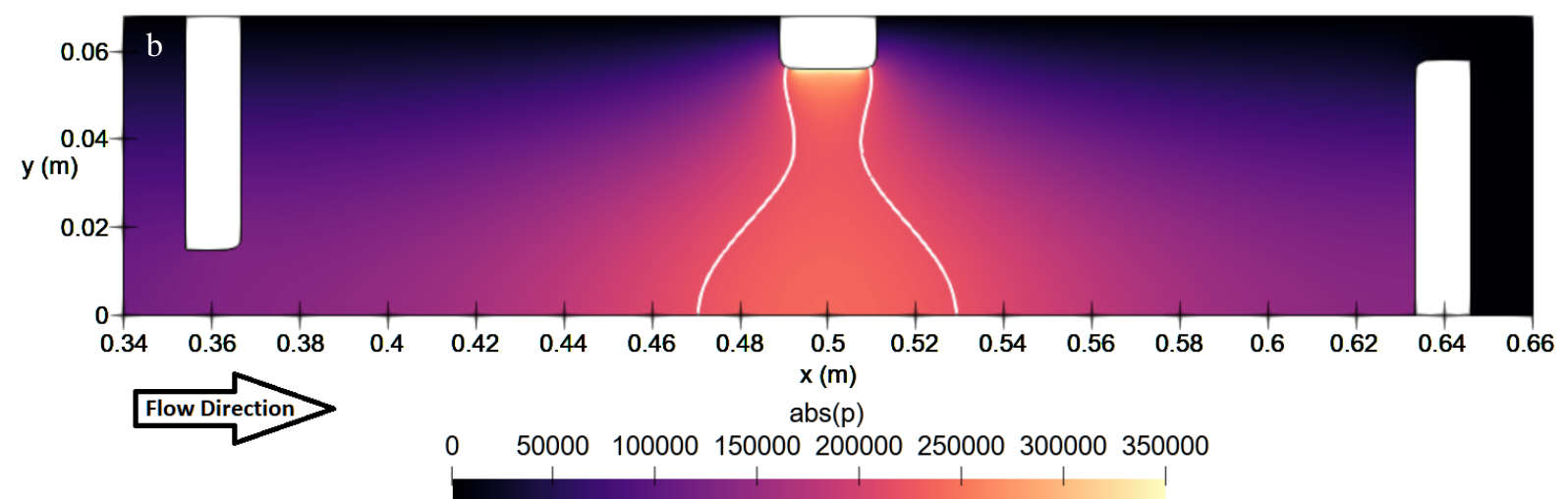

Figure 2. Acoustic pressure simulations with inlet temperatures of $950 \mathrm{~K}$ (a) and $980 \mathrm{~K}$ (b) with the sonotrode operating at $20 \mu \mathrm{m}$ peak-to-peak displacement. The white highlighted region under the sonotrode indicates a region above the Blake threshold, where intense inertial cavitation activity is expected.

The resulting acoustic streaming did not significantly change with temperature, at least within the tested range. However, the decreased viscosity at higher temperatures can result in larger secondary vortices due to the increased Reynolds number, as seen in Figure 3. This was particularly noticeable when using a $20 \mu$ m sonotrode displacement, due to the reduced streaming. This can been seen by comparing the resulting flow field for a 950 $\mathrm{K}$ inlet temperature (Figure 3a), and $980 \mathrm{~K}$ inlet temperature (Figure 3b), where the size of the secondary vortex behind the upstream partition can be seen to slightly increase.
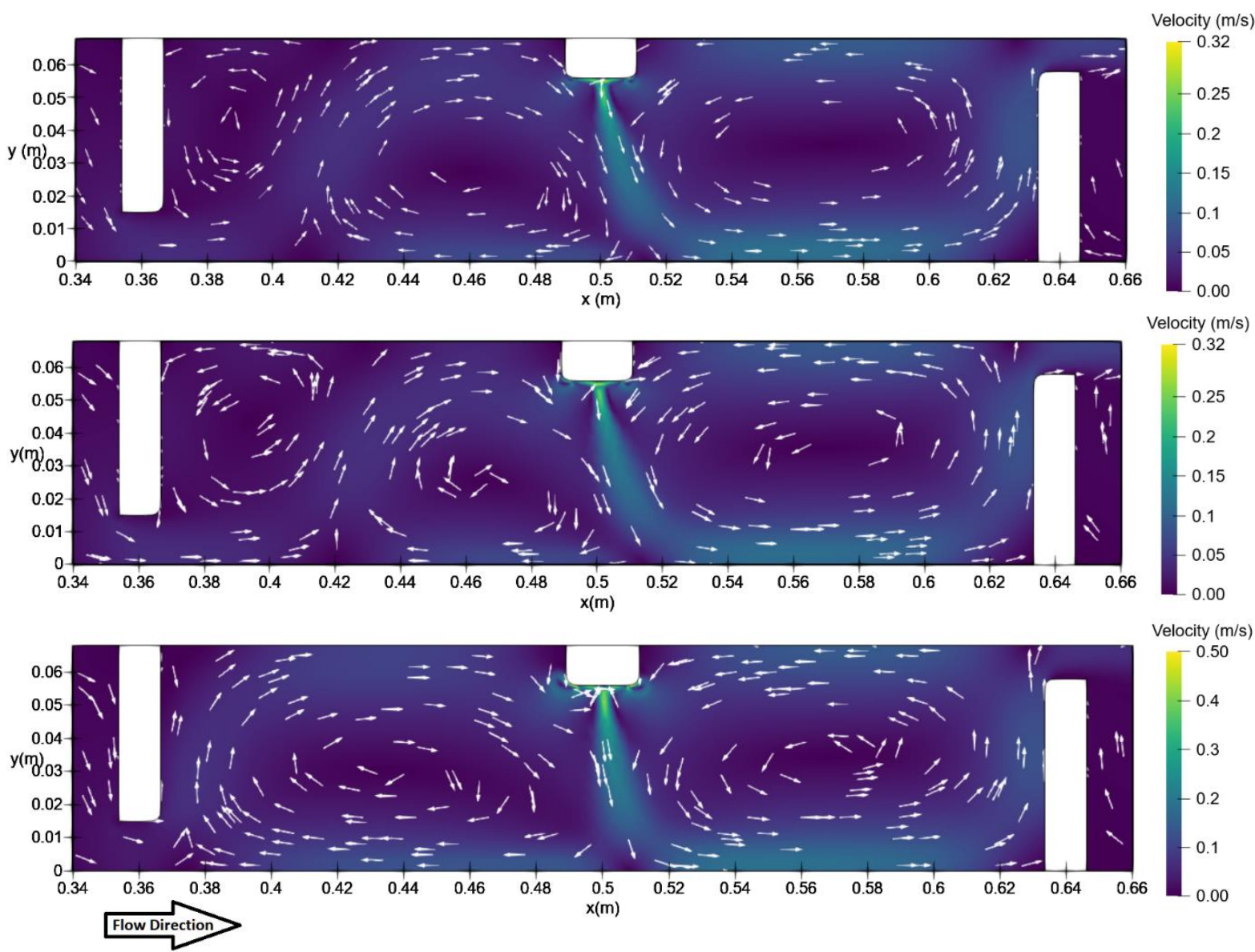

Figure 3. Fluid flow field using (top) a $20 \mu \mathrm{m}$ sonotrode displacement with an inlet temperature of $950 \mathrm{~K}$, (middle) the same sonotrode displacement but with an inlet temperature of $980 \mathrm{~K}$, and (bottom) with a $950 \mathrm{~K}$ inlet temperature and a sonotrode displacement of $40 \mu \mathrm{m}$. The arrows show the direction of fluid flow and are scaled with its velocity. 
Temperature profiles are given in Figure 4 for an inlet temperature of $950 \mathrm{~K}$ and a sonotrode displacement of $20 \mu \mathrm{m}$ and $40 \mu \mathrm{m}$. In both cases, recirculation causes a relatively sharp temperature gradient under the sonotrode. Additionally, the temperature between the partitions for a $965 \mathrm{~K}$ inlet temperature, $40 \mu \mathrm{m}$ case is given. The two higher power cases reach a downstream casting temperature of $919 \mathrm{~K}$ and $945 \mathrm{~K}$. Close to, but not quite the experimental casting temperatures of $923 \mathrm{~K}$ and $938 \mathrm{~K}$. Under the sonotrode, the case with an inlet temperature of $965 \mathrm{~K}$ and a sonotrode amplitude of $40 \mu \mathrm{m}$ reached between 950-952 $\mathrm{K}$ under the sonotrode, while the $950 \mathrm{~K}$ case reached a temperature of $934-936 \mathrm{~K}$.

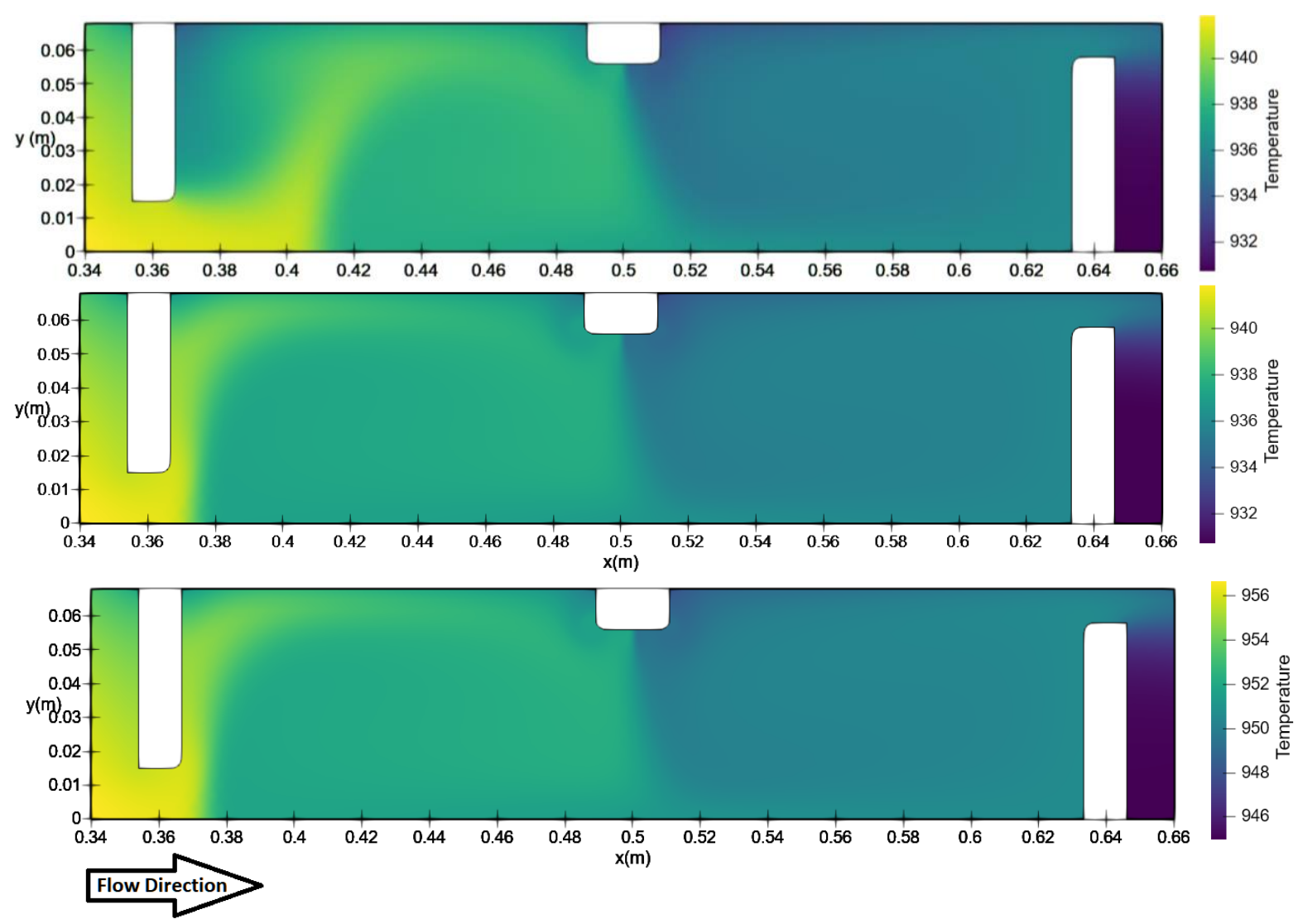

Figure 4, Temperature profiles at (a) an inlet temperature of $950 \mathrm{~K}, 20 \mu \mathrm{m}$ sonotrode displacement, (b) an inlet temperature of $950 \mathrm{~K}, 40 \mu \mathrm{m}$ sonotrode displacement, and (c) an inlet temperature of $965 \mathrm{~K}, 40 \mu \mathrm{m}$ sonotrode displacement, respectively.

A particle model was then used to estimate the residence time for each case, with particles are released at the inlet at $\mathrm{t}=0$ and collected at the outlet up until $\mathrm{t}=150 \mathrm{~s}$. As seen in Figure 5 (a), nearly all the tracer particles pass through the area of intense cavitation for all tested cases. The lowest residence time occurred in the case with an inlet temperature of $950 \mathrm{~K}$ and a sonotrode displacement of $20 \mu \mathrm{m}$, with a recorded mean of $0.33 \mathrm{~s}$. This is expected, due to the small active zone as shown in Figure 2a. The best residence times were obtained in the 980 $\mathrm{K}$ inlet, $40 \mu \mathrm{m}$ sonotrode case, with a mean residence time of $0.99 \mathrm{~s}$, suggesting that these parameters would be useful for effective processing. The $965 \mathrm{~K}$ and $950 \mathrm{~K} 40 \mu \mathrm{m}$ sonotrode cases, most closely matching the experimental data, did not seem to differ significantly in residence times, with both cases recording a mean of $0.91 \mathrm{~s}$ and $0.89 \mathrm{~s}$ respectively. However, slightly higher pressures in the active zone as seen in Figure 5 (b) could still lead to more effective treatment. 

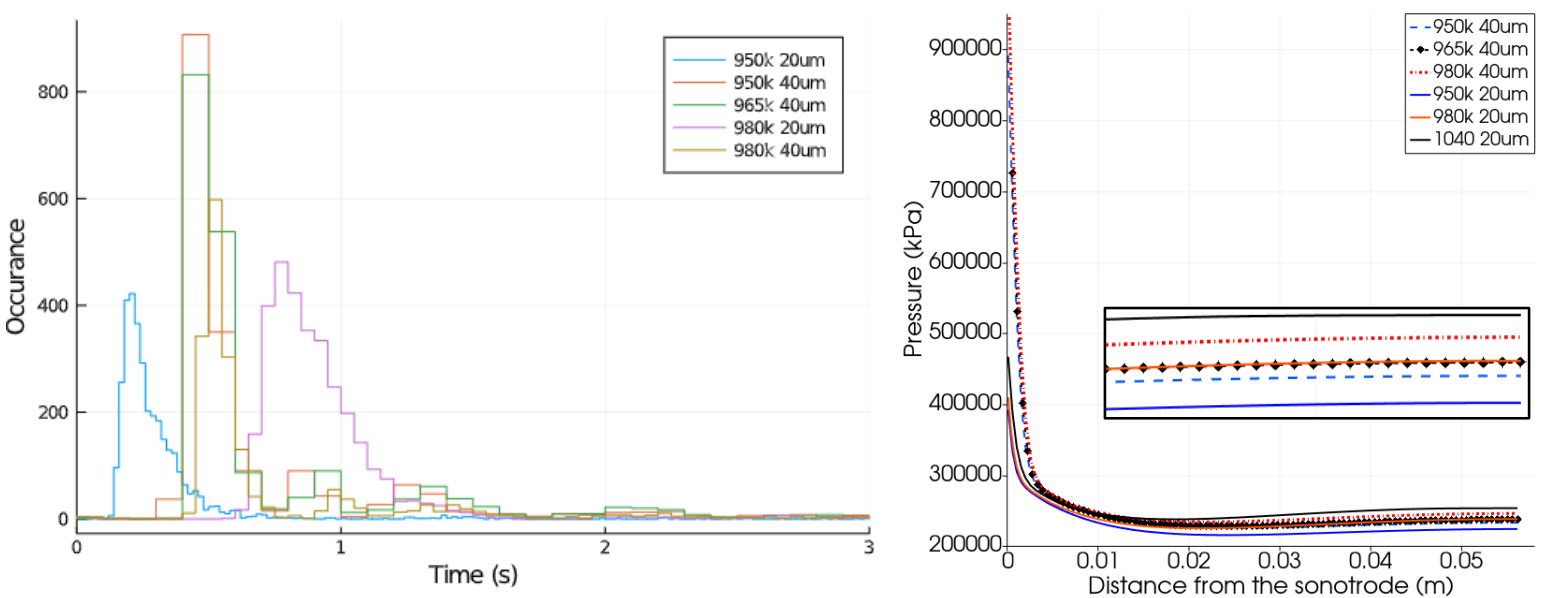

Figure 5. (a) Residence times calculated from a particle model after computing the steady state fluid velocity field. (b) Acoustic pressure magnitude in a line directly from the sonotrode. Steep attenuation of the acoustic wave at higher sonotrode displacements due to increased shielding cause an increase in acoustic streaming. Pressures far from the main cavitation zone are similar but are generally seen to increase with temperature.

\section{Experimental results}

Figure 6 shows that there are typically more $\mathrm{Al}_{3} \mathrm{Zr}$ particles in the billet cast at $938 \mathrm{~K}$ (Figure $6 \mathrm{a}$ - pointed by red arrows) than on the billet cast at $923 \mathrm{~K}$ (Figure $6 \mathrm{~b}$ - pointed by red arrows). This has been confirmed through particle number density measurements which demonstrated that the number density of $\mathrm{Al}_{3} \mathrm{Zr}$ particles almost doubled in the billet cast at $938 \mathrm{~K}(0.34)$ as compared to the billet cast at $923 \mathrm{~K}(0.18)$. This indicates that particle refinement occurs more effectively when UST was performed at higher melt temperature, closer to the liquidus of the $\mathrm{Al}_{3} \mathrm{Zr}$ phase. The numerical simulation results suggest that another contributing factor could be higher acoustic pressures and larger high-pressure regions of cavitation activity, in addition to longer residence times, when UST is performed at higher temperatures, which is also in agreement with a previous study [24].
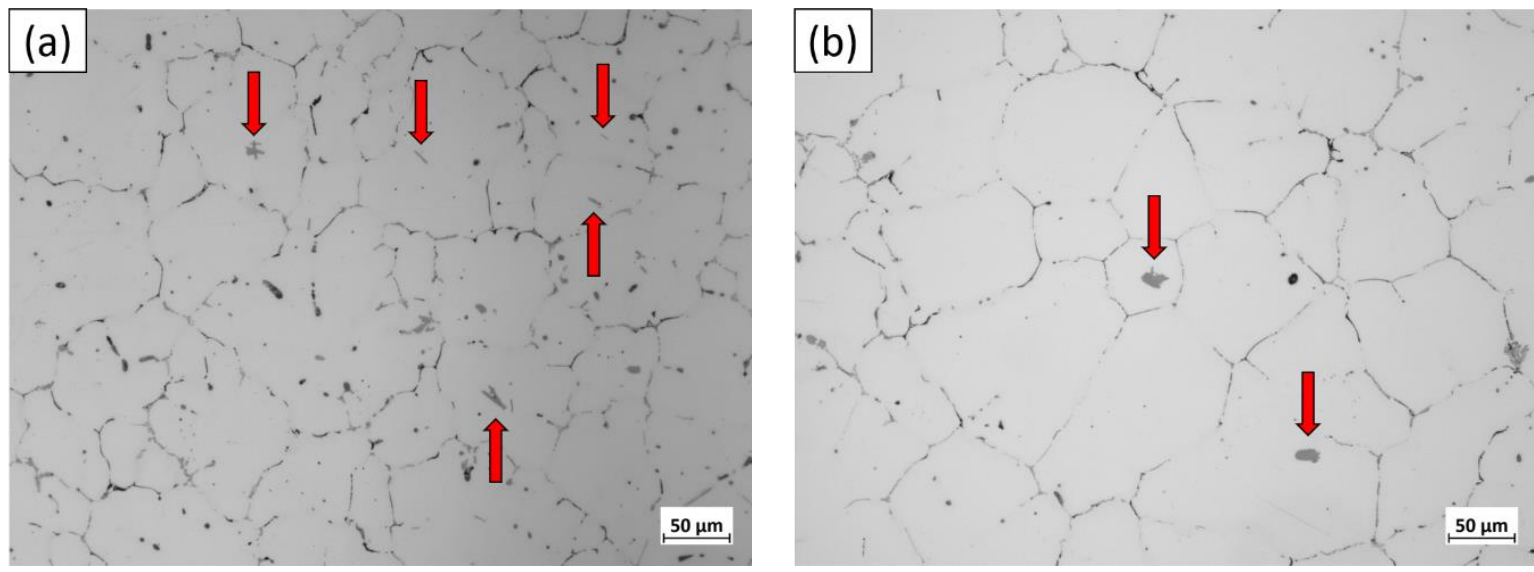

Particle number density (particles/unit area)

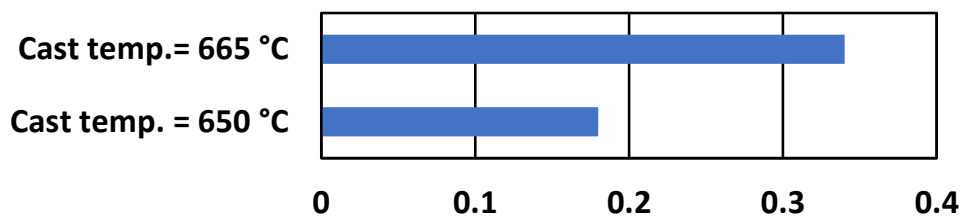

(c)

Figure 6. Optical microscope observations of $\mathrm{Al}_{3} \mathrm{Zr}$ particles in the central part of the billets cast with UST at a temperature of (a) $938 \mathrm{~K}$ or (b) $923 \mathrm{~K}$. $\mathrm{Al}_{3} \mathrm{Zr}$ particles are pointed by the red arrows. (c) Particle number density analysis at different UST conditions and casting temperatures. 
In terms of the average grain size, although in general there is not much difference between the two cast temperatures, the average grain size in the center of the billet cast at $938 \mathrm{~K}$ is marginally finer $(100 \pm 4 \mu \mathrm{m})$ as compared to its lower temperature cast counterpart $(923 \mathrm{~K}$ ) which is $110 \pm 1 \mu \mathrm{m}$. This could be explained by the number of solidification substrates being adequate, meaning the melt temperature did not play significant role, rather UST provided a major contribution in the activation and multiplication process of the potential substrate (i.e. $\mathrm{Al}_{3} \mathrm{Zr}$ ).

\section{Conclusions}

In this study, experimental results were provided showing the effect of temperature on $\mathrm{Al}_{3} \mathrm{Zr}$ particle number density in a billet after UST. Casting at a higher melt temperature of $938 \mathrm{~K}$ resulted in a billet with an increase in particle number density, and a decreased grain size, than when casting at a lower temperature of 923. Numerical simulations predict that this corresponds to a processing temperature close to $950 \mathrm{~K}$ under the sonotrode, and that one mechanism for this effect could be the reduction in hydrogen gas bubbles due to more efficient degassing and a change in hydrogen solubility, along with a decrease in the Blake threshold due to reduced surface tension. The decreased grain size observed in experiments may also be due to increased residence time, as numerical simulations predict that higher temperatures result in larger active processing areas where the particle fragmentation is likely to take place. Sonotrode power was shown to significantly affect the magnitude of acoustic streaming, increasing the flow velocity and altering the residence time. The per-bubble contribution to attenuation through viscous losses, thermal losses, or acoustic radiation, did not change significantly as the temperature changed, except for a small shift in line with the change in the Blake threshold, which suggests the primary factor for any differences in the acoustic field came from changes in the quantity of hydrogen bubbles in the system. The findings from this study suggest that performing UST at a sufficiently high temperature (still close or below to the liquidus of the $\mathrm{Al}_{3} \mathrm{Zr}$ phase) could be more beneficial for the resulting cast.

\section{Acknowledgements}

Financial support from EPSRC (UK) under the Ultramelt2 project (EP/R011001/1, EP/R011044/1, and EP/R011095/1)

\section{References}

1. Eskin GI, Eskin DG (2015) Ultrasonic Treatment of Light Alloy Melts, 2nd ed. CRC Press

2. Eskin DG, Tzanakis I, Wang F, et al (2019) Fundamental studies of ultrasonic melt processing. Ultrasonics Sonochemistry 52:455-467. https://doi.org/10.1016/j.ultsonch.2018.12.028

3. Lebon GSB, Salloum-Abou-Jaoude G, Eskin D, et al (2019) Numerical modelling of acoustic streaming during the ultrasonic melt treatment of direct-chill (DC) casting. Ultrasonics Sonochemistry 54:171-182. https://doi.org/10.1016/j.ultsonch.2019.02.002

4. Liu Z, Li R, Jiang R, et al (2019) Scalable Ultrasound-Assisted Casting of Ultra-large 2219 Al Alloy Ingots. Metall Mater Trans A 50:1146-1152. https://doi.org/10.1007/s11661-018-5097-y

5. Wang F, Qiu D, Liu Z-L, et al (2013) The grain refinement mechanism of cast aluminium by zirconium. Acta Materialia 61:5636-5645. https://doi.org/10.1016/j.actamat.2013.05.044

6. Murty BS, Kori SA, Chakraborty M (2002) Grain refinement of aluminium and its alloys by heterogeneous nucleation and alloying. International Materials Reviews 47:3-29. https://doi.org/10.1179/095066001225001049

7. Atamanenko TV, Eskin DG, Sluiter M, Katgerman L (2011) On the mechanism of grain refinement in Al-Zr-Ti alloys. Journal of Alloys and Compounds 509:57-60. https://doi.org/10.1016/j.jallcom.2010.09.046

8. Atamanenko TV, Eskin DG, Zhang L, Katgerman L (2010) Criteria of Grain Refinement Induced by Ultrasonic Melt Treatment of Aluminum Alloys Containing Zr and Ti. Metall and Mat Trans A 41:20562066. https://doi.org/10.1007/s11661-010-0232-4

9. Sreekumar VM, Eskin DG (2016) A New Al-Zr-Ti Master Alloy for Ultrasonic Grain Refinement of Wrought and Foundry Aluminum Alloys. JOM 68:3088-3093. https://doi.org/10.1007/s11837-016-2120$\mathrm{X}$ 
10. Easton MA, StJohn DH (2001) A model of grain refinement incorporating alloy constitution and potency of heterogeneous nucleant particles. Acta Materialia 49:1867-1878. https://doi.org/10.1016/S13596454(00)00368-2

11. Priyadarshi A, Khavari M, Subroto T, et al (2021) On the governing fragmentation mechanism of primary intermetallics by induced cavitation. Ultrasonics Sonochemistry 70:105260. https://doi.org/10.1016/j.ultsonch.2020.105260

12. Wang F, Eskin D, Connolley T, Mi J (2017) Influence of ultrasonic treatment on formation of primary Al $3 \mathrm{Zr}$ in $\mathrm{Al}-0.4 \mathrm{Zr}$ alloy. Transactions of Nonferrous Metals Society of China 27:977-985. https://doi.org/10.1016/S1003-6326(17)60115-8

13. Trujillo FJ (2018) A strict formulation of a nonlinear Helmholtz equation for the propagation of sound in bubbly liquids. Part I: Theory and validation at low acoustic pressure amplitudes. Ultrasonics Sonochemistry 47:75-98. https://doi.org/10.1016/j.ultsonch.2018.04.014

14. Louisnard O (2012) A simple model of ultrasound propagation in a cavitating liquid. Part I: Theory, nonlinear attenuation and traveling wave generation. Ultrasonics Sonochemistry 19:56-65. https://doi.org/10.1016/j.ultsonch.2011.06.007

15. Subroto T, Eskin DG, Beckwith C, et al (2020) Improving Ultrasonic Melt Treatment Efficiency Through Flow Management: Acoustic Pressure Measurements and Numerical Simulations. In: Tomsett A (ed) Light Metals 2020. Springer International Publishing, Cham, pp 981-987

16. Eskin DG (2017) Ultrasonic processing of molten and solidifying aluminium alloys: overview and outlook. Materials Science and Technology 33:636-645. https://doi.org/10.1080/02670836.2016.1162415

17. Lebon GSB, Pericleous K, Tzanakis I, Eskin D (2016) A model of cavitation for the treatment of a moving liquid metal volume. International Journal of Cast Metals Research 29:324-330. https://doi.org/10.1080/13640461.2016.1165460

18. Subroto T, Eskin DG, Beckwith C, et al (2020) Structure Refinement Upon Ultrasonic Melt Treatment in a DC Casting Launder. JOM. https://doi.org/10.1007/s11837-020-04269-3

19. Keller JB, Miksis M (1980) Bubble oscillations of large amplitude. The Journal of the Acoustical Society of America 68:628-633. https://doi.org/10.1121/1.384720

20. DifferentialEquations.j1 - A Performant and Feature-Rich Ecosystem for Solving Differential Equations in Julia. https://openresearchsoftware.metajnl.com/article/10.5334/jors.151/. Accessed 8 Sep 2020

21. Harkin A, Nadim A, Kaper TJ (1999) On acoustic cavitation of slightly subcritical bubbles. Physics of Fluids 11:274-287. https://doi.org/10.1063/1.869878

22. Xu H, Han Q, Meek TT (2008) Effects of ultrasonic vibration on degassing of aluminum alloys. Materials Science and Engineering: A 473:96-104. https://doi.org/10.1016/j.msea.2007.04.040

23. Thermophysical Properties of Liquid Aluminum | SpringerLink. https://link.springer.com/article/10.1007/s11661-017-4053-6. Accessed 6 Sep 2020

24. Lebon GSB, Tzanakis I, Pericleous K, Eskin D (2018) Experimental and numerical investigation of acoustic pressures in different liquids. Ultrasonics Sonochemistry 42:411-421. https://doi.org/10.1016/j.ultsonch.2017.12.002 